\title{
The clinical implication of hepatic venous territory mapping in living donor liver transplantation using right liver graft
}

\author{
Jinsoo Rhu ${ }^{1}$, Jae-Won Joh ${ }^{1}$, Woo Kyeong Jeong ${ }^{2}$, Gyu-Seong Choi ${ }^{1}$, Jong Man Kim ${ }^{1}$ \\ ${ }^{1}$ Department of Surgery-Transplantation, Samsung Medical Center, Seoul, Korea \\ ${ }^{2}$ Department of Radiology, Samsung Medical Center, Seoul, Korea
}

Background: We designed this study to evaluate the clinical implication of hepatic venous territory mapping in living donor liver transplantation.

Methods: Living donor liver transplantation cases performed using right liver graft since 2017 were included. Hepatic venous mapping using volume viewer application in the AW server 3.2 (GE Healthcare, Chicago, IL, USA) was started since January 2019. Comparison between transplantation cases with venous mapping and cases without mapping were performed. Among patients with hepatic venous mapping, cut-off point for graft occlusion were analyzed.

Results: Among 754 patients included to the study, 213 patients underwent hepatic venous mapping. Inferior hepatic vein reconstruction rate and patency rate were similar between the no mapping group $(25.2 \%$ vs. $28.8 \% ; \mathrm{P}=0.402)$ and mapping group $(92.5 \%$ vs. $96.8 \% ; P=0.412)$, respectively. While middle hepatic vein reconstruction rate was higher in the mapping group $(67.3 \%)$ compared to the no mapping group $(55.8 \%)(P=0.013)$. However, patency rate was higher in the no mapping group $(63.5 \%)$ compared to the mapping group $(51.1 \%)(\mathrm{P}=0.041)$. In patients with $\mathrm{V} 5$ reconstruction, median volume $\left(177 \mathrm{~cm}^{3}\right.$; interquartile range [IQR], 152-259 vs. $147 \mathrm{~cm}^{3}$; IQR, 113-199) ( $\mathrm{P=0.006)}$ and median percentage of V5 territory (22.3\%; IQR, 17.1-29.7 vs. 18.4\%; IQR, 14.9-21.8) $(P=0.001)$ were higher in the patent graft compared to occluded graft. A cut-off point of $150 \mathrm{~cm}^{3}$ (sensitivity, 0.824 ; specificity, 0.533; area under the receiver operating characteristic $[R O C]$ curve $[A U C]=0.680 ; P=0.006$ ) and $20.0 \%$ (sensitivity, 0.647; specificity, 0.711; AUC $=0.716 ; \mathrm{P}=0.001$ ) were chosen based on Youden index in AUC-ROC analysis.

Conclusions: Hepatic venous mapping provided objective measure for performing venous outflow reconstruction in living donor liver transplantation using right liver graft with increased reconstruction rate of the middle hepatic vein territory.

Corresponding author: Jae-Won Joh

E-mail: jw.joh@samsung.com

(c) The Korean Society for Transplantation

This is an Open Access article distributed under the terms of the Creative Commons Attribution Non-Commercial License (http://creativecommons.org/licenses/by-nc/4.0/) which permits unrestricted non-commercial use, distribution, and reproduction in any medium, provided the original work is properly cited. 